\title{
Estructura anatómica de la madera de dos
}

\section{encinos de Oaxaca}

\section{Anatomical structure of the wood of two oaks from Oaxaca, Mexico}

\author{
Faustino Ruiz-Aquinol, ${ }^{3}$, Marcos M. González-Peña2*, Juan I. Valdez-Hernández³ y Angélica Romero-Manzanares
}

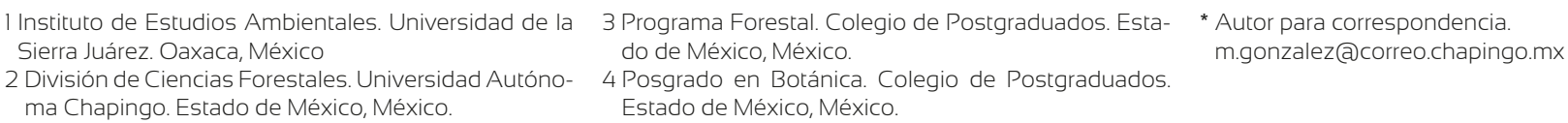

\section{RESUMEN}

México es un centro de distribución para las especies del género Quercus, con 161 especies registradas, de las que 60 presentan características favorables para el aprovechamiento maderable. Aquí se describen las características anatómicas de la madera de Quercus laurina y Q. crassifolia, y se correlacionan con su densidad básica, contracción volumétrica y dureza. La madera de $Q$. laurina presentó brillo alto y veteado medio, mientras que en Q. crassifolia fueron medio y pronunciado, respectivamente. Ambas especies presentan porosidad anular, poros solitarios de forma oval, radios uniseriados, multiseriados y agregados, parénquima apotraqueal difuso en agregados, y fibras libriformes y fibrotraqueidas. En Q. laurina se determinó una correlación entre el espesor de pared de las fibras y la densidad básica $(\mathrm{r}=0,97)$, y entre el diámetro de lumen de las fibras y la contracción volumétrica $(\mathrm{r}>0,99)$. En Q. crassifolia, las correlaciones más altas se dieron entre la contracción volumétrica y el espesor de pared de las fibras $(\mathrm{r}=0,97)$, y con el diámetro de las fibras $(\mathrm{r}=0,93)$. En los dos encinos, la contracción volumétrica alta y la presencia de radios muy anchos, indican una mayor propensión de la madera a presentar grietas y rajaduras durante el proceso de secado.

Palabras clave: contracción volumétrica, densidad básica, dureza, Quercus crassifolia, Quercus laurina.

\section{ABSTRACT}

Mexico is a distribution center for the genus Quercus, with 161 species recorded; some 60 of these have favorable characteristics for lumber usage. Herein, we describe the anatomical characteristics of Quercus crassifolia and Q. laurina wood, and these characteristics are correlated with wood basic density, volumetric shrinkage, and hardness. Quercus laurina wood displayed high brightness and medium grain, while in Q. crassifolia these were medium and coarse, respectively. Wood from both species was ring-porous, with solitary oval pores; parenchyma was apotracheal diffuse-in-aggregates, and there were uniseriate, multiseriate and aggregate rays, as well as libriform fibers and fibrotracheids. In Q. laurina, strong correlations were found between the fiber wall thickness and basic density $(r=0,97)$, and between the lumen diameter and volumetric shrinkage $(r>0,99)$; while in Q. crassifolia the strongest correlations occurred between the volumetric shrinkage and both the fiber wall thickness $(r=0,97)$, and the fiber diameter $(r=0,93)$. In both oaks, a high volumetric shrinkage and the presence of very broad rays, indicate a high propensity of wood to develop cracks and splits upon drying.

KEYWORDS: volumetric shrinkage, basic density, hardness, Quercus crassifolia, Quercus laurina.

\section{INTRODUCCIÓN}

La familia Fagaceae comprende 8-10 géneros y más de 900 especies (Kremer et al., 2012), donde el género Quercus presenta la mayor diversidad en la familia, con cerca de 400 especies (Aldrich y Cavender, 2011). En el continente americano se estiman 250 especies de encino (Oh y
Manos, 2008), de las que 161 se han registrado en México (Valencia, 2004); de esas especies, al menos 60 presentan características favorables para el aprovechamiento maderable en México (Quintanar, 2002). La mayoría de los encinos en México se encuentran en zonas montañosas con clima templado o templado subhúmedo, y más de 
95\% de las especies se encuentran entre los $1200 \mathrm{~m}$ snm y 2800 m snm (Rzedowski, 1978); la mayor riqueza de especies de Quercus se concentra en las montañas del sur (Nixon, 1993).

El predio comunal de Ixtlán de Juárez, Oaxaca, comprende $193,1 \mathrm{~km}^{2}$, de los que $64,2 \%$ son de bosque templado, y donde Quercus laurina Humb. \& Bonpl. y Q. crassifolia Humb. \& Bonpl. cohabitan con Arbutus xalapensis Kunth, Alnus acuminata arguta (Schltdl.) Furlow y Prunus serotina Ehrn., entre otras latifoliadas, y con Pinus spp. (Aquino et al., 2012; Ruiz-Aquino et al., 2015a). En este bosque, coexisten 416 árboles por hectárea de Q. laurina y 419 árboles por hectárea de Q. crassifolia; en su mayoría con fustes rectos, limpios y cilíndricos (Ruiz-Aquino et al., 2014).

Quercus laurina (subgénero Erytrobalanus), es un árbol de $10 \mathrm{~m}$ a $25 \mathrm{~m}$ de alto, tiene hojas deciduas, anchamente lanceoladas u oblanceoladas, de $7 \mathrm{~cm}$ a $10 \mathrm{~cm}$ de largo por 2,5 $\mathrm{cm}$ a $3 \mathrm{~cm}$ de ancho; su fruto es una bellota ovoide de $12 \mathrm{~mm}$ de largo (De la Paz Pérez et al., 2006). Se distribuye a lo largo de la Sierra Madre Occidental, de la Sierra Madre del Sur y por la región occidental del cinturón volcánico, entre los (2000-2440) m snm y (30653300) m snm (González et al., 2004; De la Paz Pérez y Dávalos, 2008). La madera de Q. laurina presenta grandes posibilidades de ser utilizada industrialmente, debido a su buen desempeño en el maquinado (Flores et al., 2007; Flores et al., 2013). Los árboles de Q. crassifolia (subgénero Erytrobalanus) alcanzan alturas de hasta $23 \mathrm{~m}$, tienen hojas coriáceas, ovadas, obovadas o elípticas, de $7 \mathrm{~cm}$ a $17,5 \mathrm{~cm}$ de largo por $4,1 \mathrm{~cm}$ a $11,5 \mathrm{~cm}$ de ancho; su fruto es bianual, con cúpulas hemisféricas de $6 \mathrm{~mm}$ a $10 \mathrm{~mm}$ de largo (Valencia et al., 2002). Se distribuye en la Sierra Madre Occidental al NO de México, la Sierra Madre del Sur, y a lo largo del Eje Neovolcánico (Tovar y Oyama, 2004), de 600 m snm a $2850 \mathrm{~m}$ snm (Coombes, 2012). Por la respuesta de su madera en operaciones de maquinado, puede emplearse en la elaboración de molduras, lambrines, muebles y parquet (De la Paz Pérez y Dávalos, 2008; Flores et al., 2013).

\section{OBJETIVOS}

Los objetivos del presente estudio fueron determinar las características anatómicas de la madera de Q. laurina y Q. crassifolia proveniente de Ixtlán de Juárez, e identificar las diferencias entre las dos especies. También se evaluó la influencia de la estructura anatómica en la densidad, la contracción volumétrica, y la dureza de la madera.

\section{MATERIALES Y MÉTODOS}

\section{Área de estudio y selección de los árboles}

Se utilizaron árboles provenientes de dos rodales con dominancia de especies del género Quercus en el bosque templado de Ixtlán de Juárez, Oaxaca. El primer rodal,

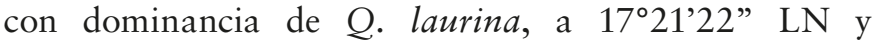
$96^{\circ} 27^{\prime} 42$ " LO, a una altitud de $2707 \mathrm{~m} \mathrm{snm}$. El segundo rodal, con dominancia de Q. crassifolia, a $17^{\circ} 21^{\prime} 08^{\prime \prime} \mathrm{LN}$ y $96^{\circ} 27^{\prime} 31^{\prime \prime}$ LO, a una altitud $2631 \mathrm{~m}$ snm. Se seleccionaron tres árboles para cada especie, sanos y representativos, con un diámetro a 1,3 m sobre el nivel del piso entre $30 \mathrm{~cm}$ y $40 \mathrm{~cm}$, y de $20 \mathrm{~m}$ a $25 \mathrm{~m}$ de altura. El derribo de los árboles y el troceo se hizo de acuerdo con Ramos y Díaz (1981), tomando para el estudio microscópico una rodaja de $5 \mathrm{~cm}$ de espesor a la altura de $1,30 \mathrm{~m}$, y para la caracterización macroscópica, la siguiente rodaja, de 30 $\mathrm{cm}$ de espesor. El material restante de los árboles derribados se utilizó para cuantificar la biomasa aérea (RuizAquino et al., 2014), y para la caracterización química y energética de la madera y el carbón de los dos encinos (Ruiz-Aquino et al., 2015b). El depósito e identificación de los especímenes se realizó en el herbario de la Universidad de la Sierra Juárez, Oaxaca.

\section{Caracterización macroscópica}

La descripción macroscópica se hizo con tablillas de $1 \mathrm{~cm}$ x $7 \mathrm{~cm}$ x $15 \mathrm{~cm}$, orientadas en los tres planos (De la Paz Pérez y Dávalos, 2008), utilizando la clasificaciones de Tortorelli (1956) para textura, porosidad, hilo y veteado, y de Ortega et al. (1988) para el brillo, olor y sabor; para la descripción del color, se usaron las tablas Munsell (1990). 


\section{Caracterización microscópica}

De la rodaja para el estudio microscópico se cortaron cubos de $2 \mathrm{~cm}$ por lado (10 cubos de albura y 10 de duramen), los que se ablandaron saturándolos al vacío con agua destilada y luego calándolos en agua hirviendo por $10 \mathrm{~min}$. Se cortaron secciones de $20 \mu \mathrm{m}$ en los tres planos con un xilotomo SM2000R (Leica, Alemania), y se tiñeron con Pardo de Bismarck a 1\% por 6 h. Luego se deshidrataron en una serie de alcoholes y finalmente se aclararon en xilol por $2 \mathrm{~min}$; las secciones se montaron con resina sintética (Sandoval, 2005). Para obtener el material disociado, se cortaron astillas y se digirieron en la solución de Franklin (1946) a $60^{\circ} \mathrm{C}$ por 24 h. Luego se enjuagaron y se tiñeron con Pardo de Bismarck, para hacer preparaciones temporales. Las mediciones se realizaron en un microscopio de luz DM 500, con cámara integrada ICC50 (Leica, Alemania). Los vasos se clasificaron en vasos con diámetro ancho $(>150 \mu \mathrm{m})$ y vasos con diámetro angosto $(\leq 150 \mu \mathrm{m})$ (Chávez et al., 2010). Se midió el diámetro mayor de los vasos en el corte transversal ( $\mathrm{n}=600$ por especie), y la longitud de elementos de vaso en el material disociado ( $\mathrm{n}=90$ por especie); las mediciones se realizaron de acuerdo con IAWA (1989). También se realizaron 120 mediciones por especie de la longitud y ancho de las fibras, del diámetro de lumen y el espesor de pared de las fibras, del alto y ancho de los radios uniseriados, del ancho y número de series en radios multiseriados, y del número de vasos por milímetro cuadrado. Los elementos mensurables se clasificaron con base en la media de acuerdo a la clasificación de Chattaway (1932), IAWA (1939) y Tortorelli (1956). Para determinar la altura de los radios multiseriados, se midieron con un vernier 32 radios completos en la albura y en el duramen de cada especie, utilizando las tablillas de la caracterización macroscópica, sin hacer distinción del árbol al que pertenecían.

Para el estudio al microscopio electrónico de barrido, se cortaron cubos de madera de $5 \mathrm{~mm}$ por lado, se deshidrataron en una serie etanólica creciente, y se recubrieron con oro. Se utilizó un microscopio JSM-6390 (JEOL Ltd., Japón), operando a $15 \mathrm{kV}$.

\section{Propiedades físicas y dureza}

Con el objetivo de establecer la influencia de las estructuras anatómicas sobre dos propiedades físicas y una propiedad mecánica de la madera, se utilizaron probetas de una mezcla de albura y duramen para determinar la densidad básica (DB) ( $\mathrm{n}=36$ por especie), la contracción volumétrica ( CV, $n=36)$ y la dureza $(\mathrm{n}=24)$, siguiendo la norma ASTM D 143-94 (ASTM, 2007). Estas propiedades se clasificaron de acuerdo a Sotomayor (2005), Chávez-Doldán (2007) y Dávalos y Bárcenas (1999), en el mismo orden. El material para estas determinaciones se obtuvo de la primera troza comercial de 1,20 m de longitud de cada árbol, aserrada en cuartos, para obtener polines de $7 \mathrm{~cm} \times 7 \mathrm{~cm}$ x $120 \mathrm{~cm}$ con sus tres planos típicos definidos (Najera et al., 2005).

\section{Análisis estadístico}

Para determinar diferencias entre los elementos celulares de las especies estudiadas, se realizó un análisis de varianza, seguido de un análisis de comparación de medias (Tukey, $\alpha=0,05$ ). Para determinar la relación de los elementos celulares con la densidad básica, la contracción volumétrica y la dureza de la madera, se calculó el coeficiente de correlación de Pearson (r). Los análisis estadísticos se realizaron usando el paquete estadístico SAS (SAS Institute, 1989).

\section{RESULTADOS Y DISCUSIÓN}

\section{Caracterización macroscópica}

En general, el duramen fue más oscuro que la albura, aunque no se observó una diferencia muy marcada entre el color de la albura y del duramen en las dos especies. La madera de Q. laurina fue más clara que la de Q. crassifolia, tanto en albura como en duramen (Tabla 1). El sabor amargo de la madera es una característica común en las dos especies, resultado de la cantidad de extractivos presentes como taninos y polifenoles. Honorato y Hernández (1998) cuantificaron los taninos de la madera de Q. laurina y de Q. crassifolia, $1,17 \%$ y $1,52 \%$ en el mismo orden, sin diferencias estadísticas entre las dos especies. La porosidad anular de las dos especies es similar a la 
TAвLA 1. Características anatómicas macroscópicas de la madera de Quercus laurina y Q. crassifolia.

\begin{tabular}{|c|c|c|c|}
\hline \multirow{2}{*}{\multicolumn{2}{|c|}{ Característica }} & \multicolumn{2}{|c|}{ Descripción } \\
\hline & & Quercus laurina & Quercus crassifolia \\
\hline \multirow{2}{*}{ Color } & Albura & Blanco 1OYR 8/2 & Castaño muy pálido 10YR 8/4 \\
\hline & Duramen & Gris claro 10YR 7/2 & Gris rosáceo 7.5 YR 6/2 \\
\hline Olor & & No característico & No característico \\
\hline Sabor & & Amargo & Amargo \\
\hline Brillo & & Alto & Medio \\
\hline Veteado & & Medio & Pronunciado \\
\hline Textura & & Media & Gruesa \\
\hline Hilo & & Recto & Recto \\
\hline Porosidad & & Anular & Anular \\
\hline
\end{tabular}

encontrada por De la Paz Pérez y Dávalos (2008) y tiene influencia sobre el veteado de la madera. Otras características anatómicas macroscópicas se presentan en la tabla 1.

\section{Caracterización microscópica}

\section{Radios}

Una de las características anatómicas más notable de la madera de encino es la presencia de radios multiseriados. Las especies que aquí se describen presentan radios uniseriados, multiseriados y agregados. Las dimensiones y el número de células de los radios se consignan en la tabla 2 . En las dos especies, los radios leñosos son homogéneos (Fig. 1A, 1B), formados por células procumbentes (Fig. 1C, 1D), lo que coincide con De la Paz Pérez et al. (2006) y De la Paz Pérez y Dávalos (2008). Los radios uniseriados en la madera de albura de Q. laurina son 3,5\% más altos que los de Q. crassifolia; sin embargo, no se encontraron diferencias significativas en la altura de los radios entre albura-albura, duramen-duramen y albura-duramen de ambas especies. Según la clasificación de Chattaway (1932), los radios uniseriados se clasifican como extremadamente bajos; respecto al ancho, los radios uniseriados se clasifican de extremadamente finos a muy finos (IAWA, 1939). Se encontraron diferencias estadísticas significati- vas entre albura y duramen de Q. laurina $(p=0,006)$, albura y duramen de $Q$. crassifolia $(p=0,046)$, y en albura-albura $(p=0,001)$ y en duramen-duramen $(p=$ $0,038)$ de las dos especies. Las medias halladas en el presente estudio para $Q$. laurina, en los radios uniseriados (número de células) y en los radios multiseriados (número de series), fueron mayores a los encontrados para la misma especie por De la Paz Pérez y Dávalos (2008), 11 células y 21 series. Para Q. crassifolia, De la Paz Pérez et al. (2005) encontraron radios multiseriados muy anchos $(387 \mu \mathrm{m}$, 20 series), valores menores a los que se obtuvieron en este estudio. No se encontraron diferencias significativas al comparar la altura de los radios multiseriados entre la albura-albura y el duramen-duramen de las dos especies. En Q. laurina, la altura de los radios multiseriados fue de $17,6 \mathrm{~mm} \pm 3,4 \mathrm{~mm}$ y de $13,2 \mathrm{~mm} \pm 1,5 \mathrm{~mm}$ en albura y duramen, respectivamente; mientras que en Q. crassifolia, la misma altura fue de $16,7 \mathrm{~mm} \pm 3,1 \mathrm{~mm}$ y $13,4 \mathrm{~mm} \pm$ 3,1 mm, en el mismo orden. Estas alturas son comparables con las obtenidas previamente para los radios multiseriados de Q. laurina (1,5 $\mathrm{mm}$ a 2,0 $\mathrm{mm}$ ) y Q. crassifolia (0,7 mm a 1,5 mm) por De la Paz Pérez y Dávalos (2008). Por otra parte, en cada una de las dos especies, los radios multiseriados fueron significativamente más altos en la albura que en el duramen ( $p<0,001$ en los dos casos). Las 
Tabla 2. Dimensiones y número de células de radios de la madera de Quercus laurina y Q. crassifolia.

\begin{tabular}{|c|c|c|c|c|c|c|c|c|}
\hline \multirow{3}{*}{ Dimensiones } & \multicolumn{4}{|c|}{ Quercus laurina } & \multicolumn{4}{|c|}{ Quercus crassifolia } \\
\hline & \multicolumn{3}{|c|}{ Árbol } & \multirow{2}{*}{ Media (DE) } & \multicolumn{3}{|c|}{ Árbol } & \multirow{2}{*}{ Media (DE) } \\
\hline & 1 & 2 & 3 & & 1 & 2 & 3 & \\
\hline \multicolumn{9}{|c|}{ Radios uniseriados albura } \\
\hline Altura $(\mu \mathrm{m})$ & 420,2 & 349,8 & 414,1 & $394,7(68)$ & 357,4 & 387,8 & 381,3 & $375,5(78)$ \\
\hline Ancho $(\mu \mathrm{m})$ & 12,3 & 15,6 & 14,1 & $14,0(3,7)$ & 16,6 & 15,3 & 16,7 & $16,2(3,4)$ \\
\hline No. células & 20,5 & 17,1 & 18,7 & $18,7(3,7)$ & 14,4 & 19,4 & 16,7 & $16,8(4,6)$ \\
\hline \multicolumn{9}{|c|}{ Radios uniseriados duramen } \\
\hline Altura $(\mu \mathrm{m})$ & 354,1 & 412,1 & 375,9 & $380,7(82)$ & 369,8 & 385,2 & 367,5 & $374,2(75)$ \\
\hline Ancho $(\mu \mathrm{m})$ & 16,1 & 16,4 & 15,5 & $16,0(4,1)$ & 21,4 & 15,3 & 16,8 & $17,8(5,3)$ \\
\hline No. células & 16,0 & 19,2 & 17,3 & $17,5(4,3)$ & 16,7 & 18,3 & 16,2 & $17,1(3,5)$ \\
\hline \multicolumn{9}{|c|}{ Radios multiseriados albura } \\
\hline Ancho $(\mu \mathrm{m})$ & 434,0 & 309,6 & 280,7 & $341,5(90)$ & 464,0 & 363,7 & 438,3 & $422,0(121)$ \\
\hline No. series & 30,9 & 24,6 & 21,8 & $25,7(5,6)$ & 28,2 & 21,9 & 25,1 & $25,0(6,1)$ \\
\hline \multicolumn{9}{|c|}{ Radios multiseriados duramen } \\
\hline Ancho $(\mu \mathrm{m})$ & 493,5 & 288,5 & 323,4 & 368,4 (120) & 384,4 & 409,2 & 643,0 & 478,9 (170) \\
\hline No. series & 34,9 & 22,5 & 25,0 & $27,4(7,5)$ & 23,8 & 24,2 & 31,7 & $26,6(7,0)$ \\
\hline
\end{tabular}

DE: desviación estándar

dimensiones de los radios son de las variables anatómicas que más influencia tienen en el proceso de secado, debido a que presentan una pared celular primaria flexible, poco engrosada y constituyen puntos débiles en la madera por la ausencia de la pared secundaria (Metcalfe y Chalk, 1985). En conjunción con su densidad alta, acentúan las contracciones altas en la madera, ocasionando problemas en su industrialización. Por otra parte, los radios visibles a simple vista, son un carácter anatómico valioso de la figura de la madera de encino, apreciado en la fabricación de pisos, muebles y chapas.

\section{Vasos y parénquima axial}

Con respecto a su longitud, los elementos de vaso se clasifican como medianos en ambas especies (Chattaway, 1932). En el plano transversal, los vasos presentan poros con diámetro tangencial de moderadamente pequeños a moderadamente grandes (IAWA, 1939). En la albura de ambas especies, los poros por milímetro cuadrado son escasos y en el duramen moderadamente escasos (Chattaway, 1932). La consideración del diámetro y la cantidad de poros por milímetro cuadrado es importante en las operaciones de acabado y pegado, ya que a mayor número por milímetro cuadrado, es más difícil darle un buen acabado a la madera y la adhesión de las superficies es menor.También tiene relevancia en los procesos de impregnación de la madera, pues la presencia de vasos numerosos es deseable para facilitar el movimiento y la distribución uniforme de los preservadores (Honorato, 2002). Las dimensiones de los vasos se presentan en la tabla 3.

La madera de Q. laurina presentó porosidad anular (Fig. 2A), similar a la encontrada para esta especie por De la Paz Pérez y Quintanar (2001) y De la Paz Pérez y Dávalos (2008), pero diferente a la porosidad semianular obtenida por Aguilar y Castro (2006) y De la Paz Pérez et al. 

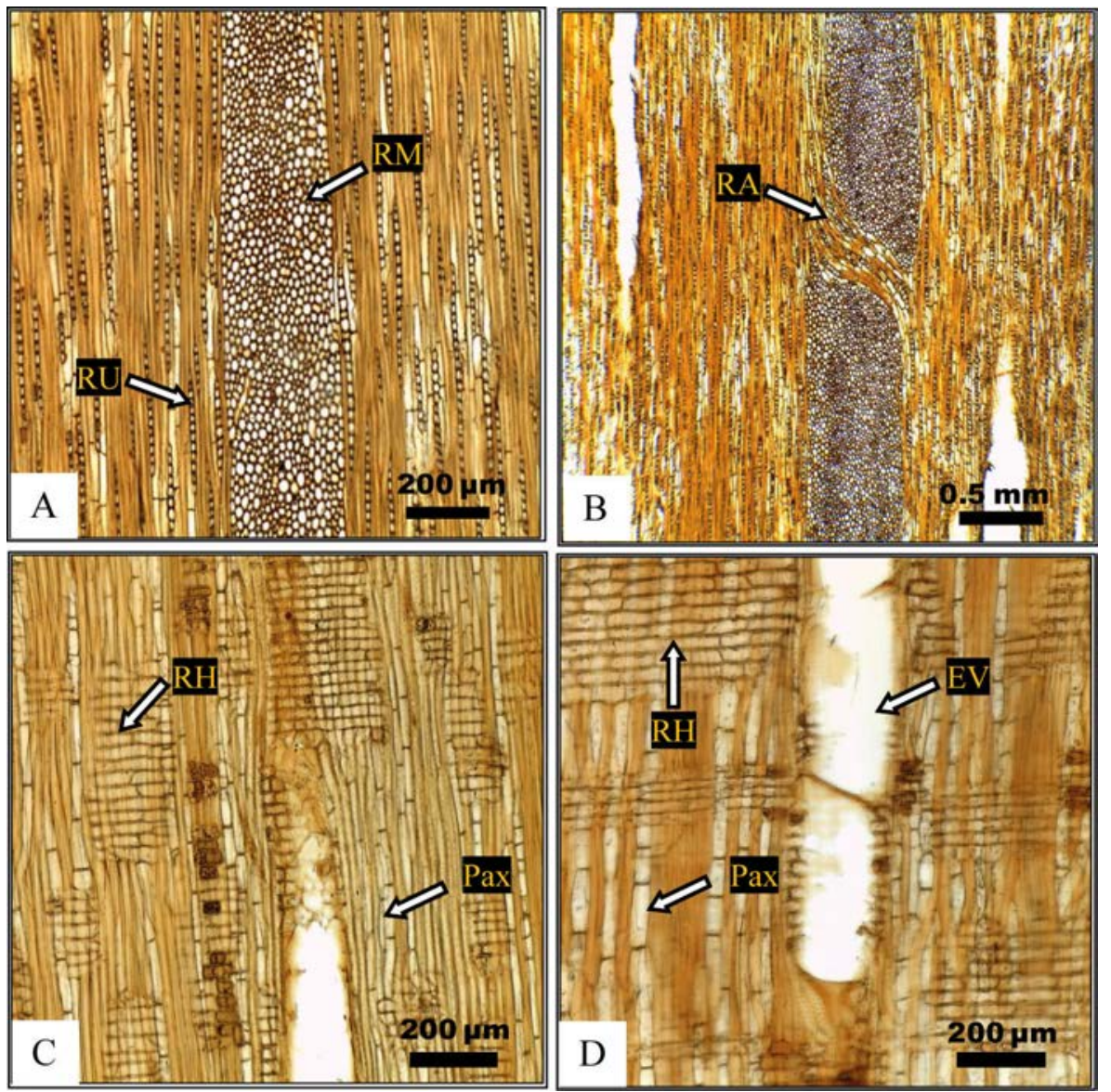

FIgURA 1. A) Quercus laurina, duramen árbol 3 (10x), RU = radio uniseriado; RM = radio multiseriado. B) Q. crassifolia, albura árbol 2 (4x); RA = radio agregado. C) Q. laurina, duramen árbol 1 (10x), RH = radio homogéneo, Pax = parénquima axial. D) Q. crassifolia, albura árbol 1 (10x), EV = elemento de vaso.

(2006). Los poros son de contorno oval y circular, solitarios y arreglados en hileras radiales, presentan tílides en la madera de duramen (Fig. 2B). Los elementos de vaso (Fig. 2C) presentan placas perforadas simples y puntuaciones areoladas alternas de forma oval (Fig. 2D), no presentan engrosamiento de la pared celular. El parénquima axial es apotraqueal difuso en agregados.

La madera de Q. crassifolia, presentó porosidad anular (Fig. 3A), lo que coincide con lo descrito para la misma especie por De la Paz Pérez y Dávalos (2008) y De la Paz Pérez y Quintanar (2001). La porosidad anular mejora las características estéticas de la madera, haciendo el veteado más atractivo. Los poros son de contorno oval y circular, solitarios y arreglados en hileras radiales y presentan tílides en la madera de duramen (Fig. 3B, Fig. 4A). Los ele- mentos de vaso presentan placas perforadas simples (Fig. 4B), y puntuaciones areoladas alternas de forma oval (Fig. 3D), con ausencia de engrosamientos de la pared celular. El parénquima axial es apotraqueal difuso en agregados. Se encontraron diferencias estadísticas entre las especies estudiadas en el número de poros por milímetro cuadrado en madera de duramen $(p=0,007)$, y en el diámetro de poros menores a $150 \mu \mathrm{m}$ en albura y duramen $(p<0,001)$.

\section{Fibras, fibrotraqueidas y traqueidas vasicéntricas}

Las dimensiones de las fibras y las características de los radios multiseriados, son las variables que más influencia tienen en las propiedades físicas y mecánicas de la madera y para establecer la factibilidad de utilizar la madera en la producción de pulpa, papel, y tableros de fibras. Las dos 

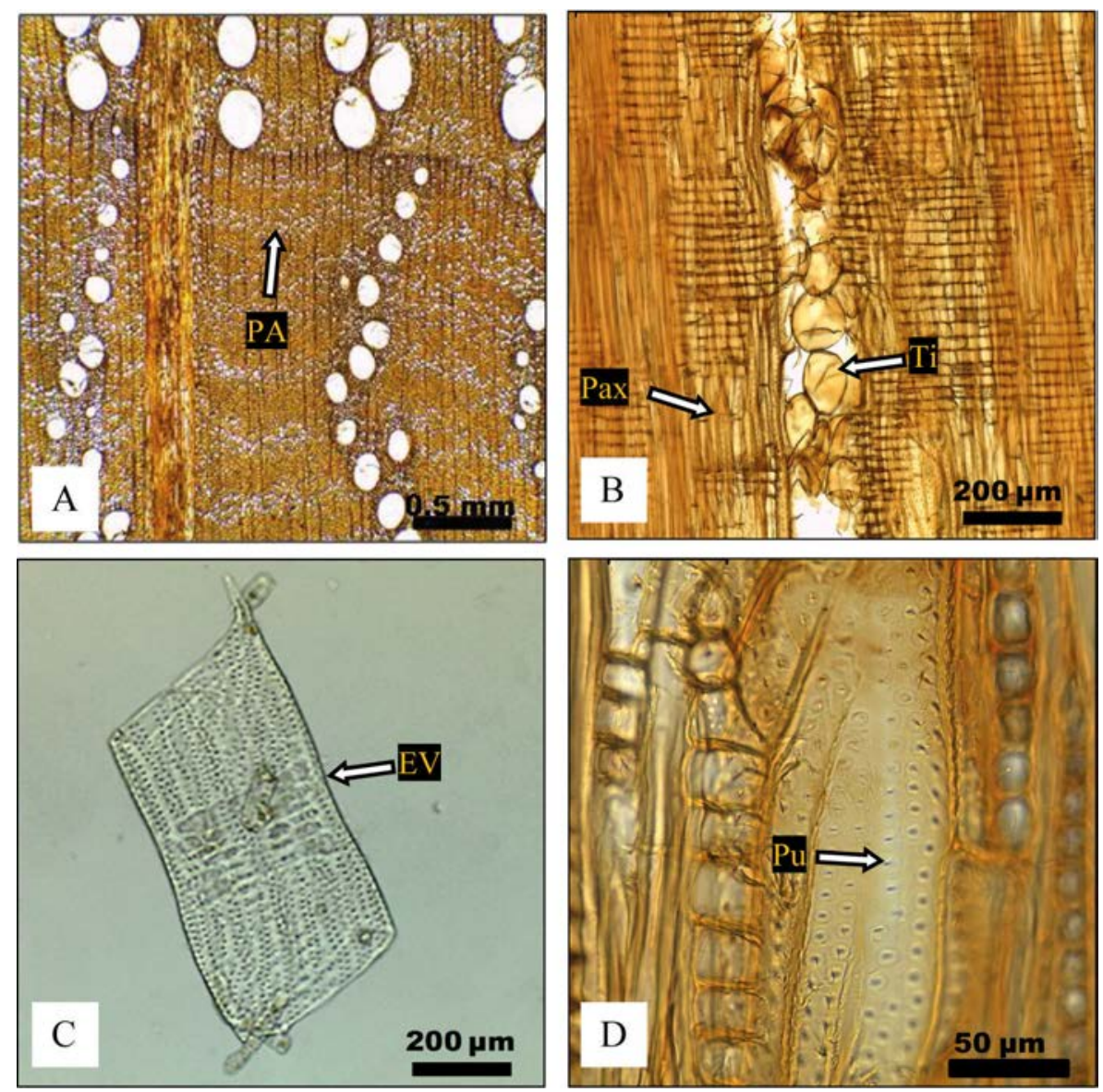

Figura 2. Quercus laurina A) albura árbol 2, porosidad anular, $\mathrm{PA}=$ parénquima apotraqueal difuso (4x). B) duramen árbol 1, Pax = parénquima axial, $\mathrm{Ti}$ = tílides $(10 \mathrm{x}) . \mathrm{C}) \mathrm{EV}=$ elemento de vaso en albura (madera temprana), material disociado árbol 1 (10x). D) Pu = puntuaciones areoladas alternas en elemento de vaso, duramen árbol 3 (40x).

especies presentan fibras de tipo libriforme, fibrotraqueidas y traqueidas vasicéntricas; en el duramen de Q. crassifolia se identificaron fibras septadas aisladas (Fig. 4D). Con respecto a la clasificación de IAWA (1937), las fibras de albura de ambas especies se clasifican como moderadamente largas, mientras que las de duramen como fibras medianas (Tabla 4). Con base en el diámetro, las fibras de albura se clasifican como medianas y las de duramen como finas; con referencia al espesor de la pared, Q. crassifolia presentó fibras de pared gruesa en albura y duramen, mientras para Q. laurina la pared es gruesa en albura y muy gruesa en duramen (Tortorelli, 1956).

Se encontraron diferencias significativas entre la longitud ( $p<0,001)$, el diámetro $(p=0,002)$ y el espesor de pared $(p<0,001)$ de fibras de albura y duramen de $Q$. laurina, y también entre la longitud $(p<0,001)$ y el diámetro $(p<0,001)$ de fibras de albura y duramen de $Q$. crassifolia. No se encontraron diferencias estadísticas significativas en los tres parámetros al comparar las fibras de albura-albura y duramen-duramen de las dos especies $(p>0,05)$.

\section{Propiedades físicas}

La densidad básica (DB) de la madera de Q. laurina fue de $0,693 \mathrm{~g} \mathrm{~cm}^{-3}$, superior a los $0,600 \mathrm{~g} \mathrm{~cm}^{-3}$ encontrados por Honorato y Fuentes (2001) para la misma especie y a los $0,652 \mathrm{~g} \mathrm{~cm}^{-3}$ de De la Paz Pérez y Dávalos (2008). En Q. crassifolia, la DB fue de $0,666 \mathrm{~g} \mathrm{~cm}^{-3}$, similar a los $0,687 \mathrm{~g}$ $\mathrm{cm}^{-3}$ hallados por De la Paz Pérez y Dávalos (2008) (Tabla 5). Las diferencias en la densidad de la madera ocurren 
Tabla 3. Dimensiones de los vasos de la madera de Quercus laurina y Q. crassifolia.

\begin{tabular}{|c|c|c|c|c|c|c|c|c|}
\hline \multirow{3}{*}{ Dimensiones } & \multicolumn{4}{|c|}{ Quercus laurina } & \multicolumn{4}{|c|}{ Quercus crassifolia } \\
\hline & \multicolumn{3}{|c|}{ Árbol } & \multirow{2}{*}{ Media (DE) } & \multicolumn{3}{|c|}{ Árbol } & \multirow{2}{*}{ Media (DE) } \\
\hline & 1 & 2 & 3 & & 1 & 2 & 3 & \\
\hline \multicolumn{9}{|c|}{ Albura } \\
\hline $\mathrm{D}(>150 \mu \mathrm{m})$ & 205,0 & 282,8 & 286,7 & $258,2(52,4)$ & 276,7 & 262,8 & 250,4 & $263,3(40,9)$ \\
\hline $\mathrm{D}(\leq 150 \mu \mathrm{m})$ & 95,8 & 100,6 & 109,3 & $101,9(21,6)$ & 135,5 & 117,1 & 113,8 & $122,1(22,6)$ \\
\hline Longitud ( $\mu \mathrm{m})$ & 426,0 & 427,2 & 487,0 & $446,7(87,6)$ & 419,2 & 524,7 & 496,2 & 480,0 (109) \\
\hline Número/mm² & 5,2 & 4,4 & 4,8 & $4,8(1,6)$ & 4,9 & 4,2 & 5,2 & $4,8(1,3)$ \\
\hline \multicolumn{9}{|c|}{ Duramen } \\
\hline $\mathrm{D}(>150 \mu \mathrm{m})$ & 201,5 & 258,7 & 257,0 & $239,1(45,6)$ & 241,4 & 255,5 & 216,3 & $237,7(35,9)$ \\
\hline $\mathrm{D}(\leq 150 \mu \mathrm{m})$ & 100,0 & 97,6 & 98,6 & $98,7(19,5)$ & 112,7 & 119,1 & 120,0 & $117,3(18,6)$ \\
\hline Longitud $(\mu \mathrm{m})$ & 420,9 & 440,8 & 417,5 & $426,4(86,7)$ & 447,8 & 481,5 & 455,0 & $461,5(86,6)$ \\
\hline Número/mm² & 4,6 & 5,3 & 5,2 & $5,2(2,6)$ & 5,6 & 5,0 & 5,9 & $5,5(1,6)$ \\
\hline
\end{tabular}

$\mathrm{DE}$ = desviación estándar; $\mathrm{D}$ = diámetro $(\mu \mathrm{m})$

Tabla 4. Dimensiones de las fibras de la madera de Quercus laurina y Q. crassifolia.

\begin{tabular}{|c|c|c|c|c|c|c|c|c|}
\hline \multirow{3}{*}{ Dimensiones } & \multicolumn{4}{|c|}{ Quercus laurina } & \multicolumn{4}{|c|}{ Quercus crassifolia } \\
\hline & \multicolumn{3}{|c|}{ Árbol } & \multirow{2}{*}{ Media (DE) } & \multicolumn{3}{|c|}{ Árbol } & \multirow{2}{*}{ Media (DE) } \\
\hline & 1 & 2 & 3 & & 1 & 2 & 3 & \\
\hline \multicolumn{9}{|c|}{ Albura } \\
\hline Longitud (mm) & 1,9 & 1,7 & 1,8 & $1,8(0,2)$ & 1,7 & 1,9 & 2,0 & $1,9(0,2)$ \\
\hline Diámetro $(\mu \mathrm{m})$ & 22,6 & 28,3 & 27,1 & $26,0(5,8)$ & 26,2 & 26,0 & 28,1 & $26,7(4,1)$ \\
\hline $\mathrm{DL}(\mu \mathrm{m})$ & 7,7 & 10,4 & 9,4 & $9,2(3,5)$ & 9,8 & 12,7 & 9,9 & 10,8 (11) \\
\hline $\operatorname{EP}(\mu \mathrm{m})$ & 7,5 & 9,0 & 8,8 & $8,4(1,9)$ & 8,2 & 6,6 & 9,1 & $8,0(5,5)$ \\
\hline \multicolumn{9}{|c|}{ Duramen } \\
\hline Longitud (mm) & 1,5 & 1,7 & 1,7 & $1,6(0,2)$ & 1,6 & 1,7 & 1,6 & $1,6(0,1)$ \\
\hline Diámetro $(\mu \mathrm{m})$ & 24,7 & 22,0 & 22,7 & $23,1(3,9)$ & 23,1 & 23,5 & 22,8 & $23,1(4,3)$ \\
\hline $\mathrm{DL}(\mu \mathrm{m})$ & 10,2 & 7,6 & 8,7 & $8,8(2,5)$ & 8,4 & 7,4 & 8,0 & $7,9(2,0)$ \\
\hline $\operatorname{EP}(\mu \mathrm{m})$ & 7,3 & 7,2 & 7,0 & $7,1(1,3)$ & 7,4 & 8,1 & 7,4 & $7,6(1,8)$ \\
\hline
\end{tabular}

$\mathrm{DL}=$ diámetro del lumen; $\mathrm{EP}=$ espesor de pared $(\mu \mathrm{m})$

por cambios en la proporción de los elementos constitutivos del xilema, como vasos, fibras y células de parénquima, y a la variación en el espesor de la pared celular del esclerénquima, atribuidas a las condiciones climáticas y de suelo de las distintas regiones donde crecen los individuos (Bernal y Terrazas, 2000; Montaño et al., 2013). La DB de las dos especies fue alta, de acuerdo con la clasificación de Sotomayor (2005). Como se esperaba de una 

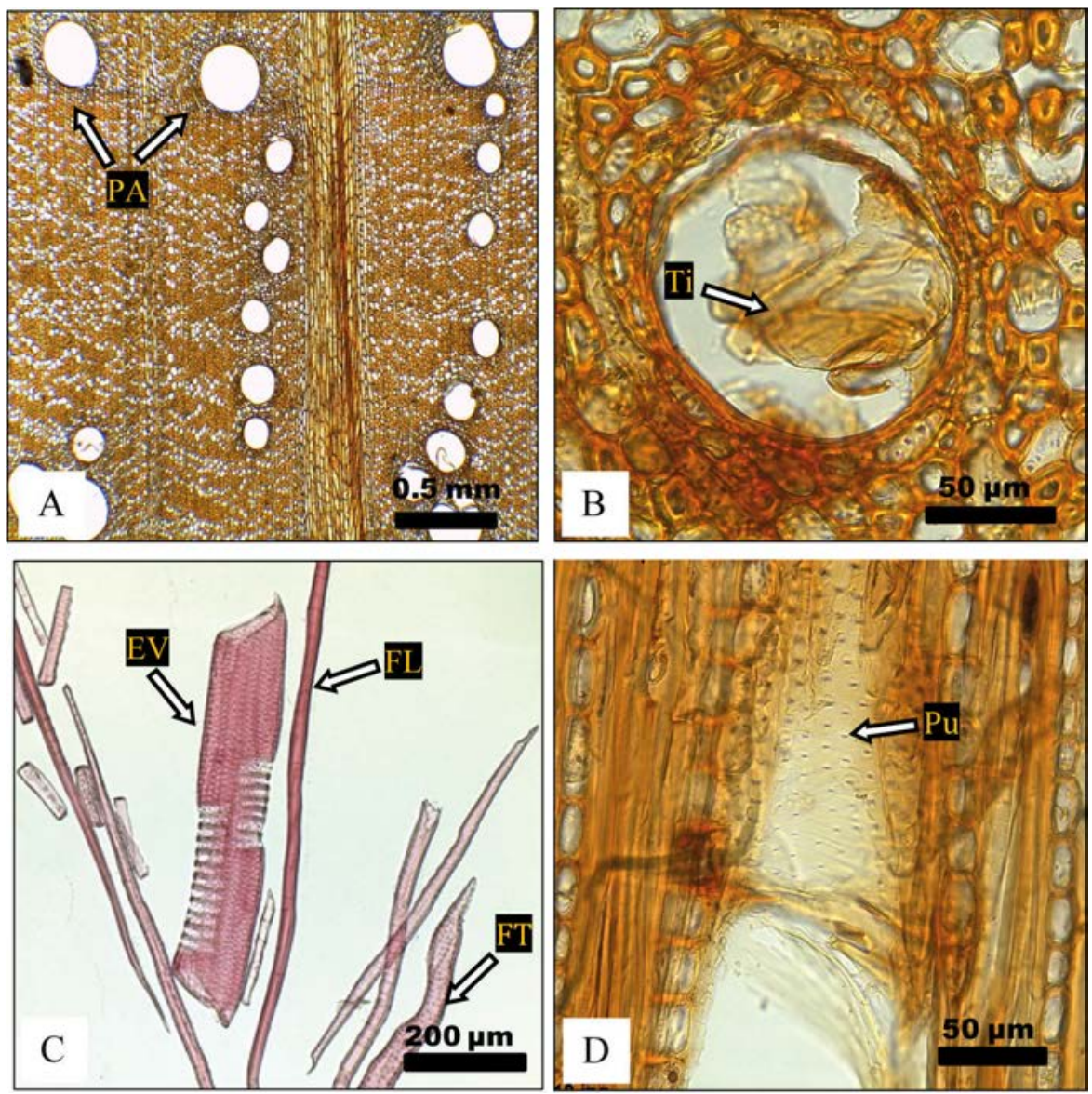

Figura 3. Quercus crassifolia, duramen árbol 2 A) porosidad anular (4x); PA = parénquima apotraqueal difuso. B) Ti = tílide, duramen árbol 3 (40x). C) EV = elemento de vaso en albura (madera tardía), material disociado árbol 3; FT = fibrotraqueida; FL = fibra libriforme (10x). D) Pu = puntuaciones areoladas alternas en elemento de vaso, albura árbol 1 (40x).

madera de densidad alta, la contracción volumétrica de las dos especies también fue alta, de acuerdo con la clasificación de Chávez-Doldán (2007). Una contracción volumétrica alta indica una menor estabilidad dimensional de la madera, lo que precisa mayor cuidado en el proceso de secado de la madera, ya que habrá una mayor tendencia a la presencia de agrietamiento y rajaduras en el material.

La dureza de la madera de Q. laurina fue superior a la de Q. crassifolia (Tabla 5) y ambas especies se clasifican como de dureza alta, de acuerdo con Dávalos y Bárcenas (1999). La dureza, como las demás propiedades físicas de la madera, están directamente relacionadas con la DB; en este estudio, Q. laurina presentó una DB superior a Q. crassifolia, lo que es consistente con los valores de dureza encontrados.

\section{Relación entre propiedades físicas y parámetros anatómicos}

De acuerdo con el análisis de correlación, se encontraron correlaciones significativas entre las propiedades físicas y algunos parámetros anatómicos (Tabla 6). Para Q. laurina, las correlaciones positivas más altas se dieron entre la densidad básica (DB) y el ancho de los radios uniseriados $(r=0,98)$ y, como se anticipaba, entre la DB y el espesor de la pared de la fibra (EPF) $(r=0,97)$. También se encontraron correlaciones positivas altas entre la contracción volumétrica y el diámetro de lumen (DL) $(\mathrm{r}=1,00)$ y entre el ancho de los radios multiseriados (ARM) y la dureza $(r=0,83)$. En Quercus crassifolia, se encontraron correlaciones positivas altas entre la DB y el DL $(r=0,99)$, 

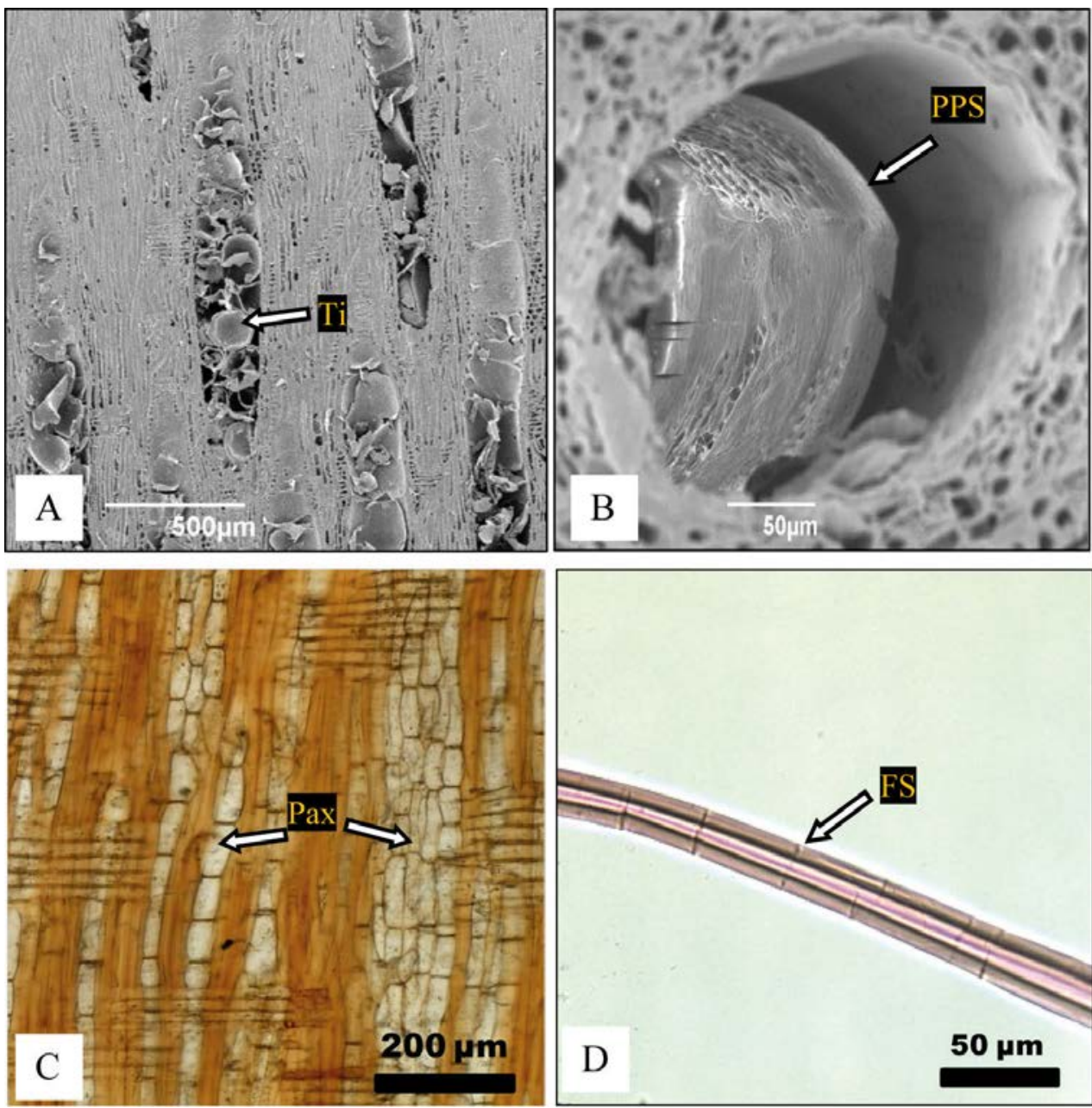

Figura 4. Quercus crassifolia. Microscopio electrónico de barrido: A) Ti = tílides en elemento de vaso, corte radial, duramen árbol 1. B) PPS = placa perforada simple, albura árbol 3. Microscopio de luz: C) Pax = parénquima axial, albura árbol 1 (10x). D) Fibra septada (FS), duramen árbol 2 (40x).

Tabla 5. Propiedades físicas de la madera de Quercus laurina y Q. crassifolia.

\begin{tabular}{|c|c|c|c|c|c|c|c|c|}
\hline \multirow{3}{*}{$\begin{array}{l}\text { Propiedades } \\
\text { físicas }\end{array}$} & \multicolumn{4}{|c|}{ Quercus laurina } & \multicolumn{4}{|c|}{ Quercus crassifolia } \\
\hline & \multicolumn{3}{|c|}{ Árbol } & \multirow{2}{*}{ Media (DE) } & \multicolumn{3}{|c|}{ Árbol } & \multirow{2}{*}{ Media (DE) } \\
\hline & 1 & 2 & 3 & & 1 & 2 & 3 & \\
\hline $\mathrm{DB}\left(\mathrm{g} \mathrm{cm}^{-3}\right)$ & 0,685 & 0,704 & 0,690 & $0,693(0,02)$ & 0,656 & 0,681 & 0,661 & $0,666(0,02)$ \\
\hline CV (\%) & 17,94 & 19,93 & 21,09 & $19,65(1,54)$ & 16,61 & 16,18 & 16,81 & $16,53(1,14)$ \\
\hline DJ (kN) & 10,10 & 8,64 & 6,90 & $8,55(2,22)$ & 7,82 & 7,37 & 9,03 & $8,08(1,13)$ \\
\hline
\end{tabular}

DE = Desviación estándar; DB = Densidad básica; CV = Contracción volumétrica; D = Dureza Janka, cara transversal (carga máxima) 
Tabla 6. Correlación entre propiedades físicas y parámetros anatómicos de la madera de Quercus laurina y Q. crassifolia.

\begin{tabular}{|c|c|c|c|c|c|c|c|c|}
\hline \multirow{2}{*}{$\begin{array}{c}\text { Propiedades } \\
\text { físicas }\end{array}$} & \multicolumn{8}{|c|}{ Coeficiente de correlación (r) } \\
\hline & $D V>150$ & $D V<150$ & $L V$ & $D F$ & EPF & $D L$ & $A R U$ & $A R M$ \\
\hline & \multicolumn{8}{|c|}{ Quercus laurina } \\
\hline Densidad básica & 0,86 & 0,19 & 0,36 & 0,94 & 0,97 & 0,58 & 0,98 & $-0,87$ \\
\hline Contracción Vol. & 0,94 & 0,89 & 0,95 & 0,85 & 0,80 & 1,00 & 0,46 & $-0,93$ \\
\hline \multirow[t]{2}{*}{ Dureza } & $-0,85$ & $-0,96$ & $-0,99$ & $-0,73$ & $-0,66$ & $-0,99$ & $-0,27$ & 0,83 \\
\hline & \multicolumn{8}{|c|}{ Quercus crassifolia } \\
\hline Densidad básica & 0,50 & $-0,36$ & 0,80 & $-0,41$ & $-0,85$ & 0,99 & $-0,79$ & $-0,69$ \\
\hline Contracción Vol. & $-0,75$ & 0,05 & $-0,14$ & 0,93 & 0,97 & $-0,99$ & 0,57 & 0,88 \\
\hline Dureza & $-0,97$ & $-0,40$ & $-0,14$ & 0,93 & 0,98 & $-0,80$ & 0,14 & 1,00 \\
\hline
\end{tabular}

DV = diámetro del vaso; $L V=$ longitud de los elementos del vaso; $D F=$ diámetro de la fibra; EPF = espesor de pared de la fibra; $D L=$ diámetro de lumen de las fibras; $\mathrm{ARU}=$ ancho de radios uniseriados; $\mathrm{ARM}=$ ancho de radios multiseriados

entre la contracción volumétrica y el EPF $(r=0,97)$ y entre la dureza y el ARM $(\mathrm{r}=1,00)$.

Cabe señalar que, junto con los vasos, las fibras presentan características distintas en las diferentes capas de las zonas de crecimiento en las angiospermas de porosidad anular como las aquí estudiadas. Sería deseable que los futuros esfuerzos en la descripción anatómica de los encinos incluyeran un análisis de las características anatómicas de la madera temprana y de la madera tardía, así como de la relación que guardan dichas características con las propiedades físicas (e.g. densidad) y mecánicas (e.g. resistencia al impacto) de la madera.

\section{CONCLUSIONES}

La madera de Q. laurina presentó una tonalidad más clara con respecto a Q. crassifolia y el atractivo veteado de estos dos encinos permite disponer su utilización en productos decorativos. La madera de las dos especies pudo diferenciarse por el brillo alto y la textura media en Q. laurina, contra un brillo medio y una textura gruesa en Q. crassifolia. Las dos especies mostraron similitud en todos sus caracteres microscópicos, aunque en Q. crassifolia se encontraron fibras septadas. Estadísticamente se determinaron diferencias significativas entre las dos espe- cies en el ancho de los radios uniseriados en albura y duramen, en el número de poros por milímetro cuadrado en el duramen y en el diámetro de poros menores a $150 \mu \mathrm{m}$ en albura y duramen. Se determinó una correlación alta entre el espesor de pared de las fibras y la densidad básica en $Q$. laurina y entre el espesor de pared de las fibras y la contracción volumétrica en Q. crassifolia. Ambas especies mostraron correlación alta entre la contracción volumétrica y el diámetro de las fibras. Por otra parte, la dureza alta indica que la madera de estas especies puede utilizarse en la fabricación de pisos de tráfico pesado. Finalmente, la alta contracción volumétrica aquí determinada, conlleva a implementar programas de secado con condiciones de temperatura y humedad rigurosamente controladas.

\section{RECONOCIMIENTOS}

Al Programa de Mejoramiento al Profesorado (Promep) por la beca otorgada al primer autor (Folio UNSIJ/001). A la Dra. Amparo Borja de la Rosa por sus sugerencias y facilidades para usar los instrumentos del Laboratorio de Anatomía y Tecnología de la Madera de la UACh. Al Ing. Gonzalo Novelo González por su apoyo en los ensayos con la máquina universal. Al Biól. Gabriel González Adame por su ayuda en la colecta e identificación del material. 


\section{REFERENCIAS}

Aguilar, S. y B.J. Castro. 2006. Anatomía de la madera de doce especies del bosque mesófilo de montaña del Estado de México. Madera y Bosques 12(1):95-115.

Aldrich, P.R. y J. Cavender B. 2011. Quercus. In: C. Kole, ed. Wild crop relatives: genomic and breeding resources, forest trees. Springer, Berlin Heidelberg. p:89-129.

Aquino, C., F. Ruiz-Aquino y M.E. Fuente. 2012. Caracterización del patrimonio natural de la comunidad de Ixtlán de Juárez: una aproximación desde el espacio territorial. In: M.E. Fuente C., F. Ruiz A. y C. Aquino V., eds. Conocimiento indígena contemporáneo y patrimonio biocultural en la Sierra Juárez de Oaxaca: Aportaciones empíricas y analíticas hacia la sustentabilidad. Universidad de la Sierra Juárez, México. p:35-59.

ASTM (American Society for Testing and Materials). 2007. ASTM D143-94. Standard Test methods for small clear specimens of timber. ASTM International, West Conshohocken, Pennsylvania $32 \mathrm{p}$.

Bernal, S. y T. Terrazas. 2000. Influencia climática sobre la variación radial de caracteres anatómicos de madera en Abies religiosa. Madera y Bosques 6(1):73-86.

Coombes, A. 2012. Quercus crassifolia. Curtis's Botanical Magazine 29(2):162-169.

Chattaway, M. 1932. Proposed standards for numerical values used in describing woods. Tropical Woods 29:20-28.

Chávez, D. M., S. Aguilar y T. Terrazas. 2010. Variación anatómica en la madera de Quercus obtusata (Fagaceae). Madera y Bosques 16(2):69-87.

Chávez-Doldán, N.S., D. Ramírez H. y M.G. Ovelar. 2007. Estudio de los cambios dimensionales de la madera de Apuleia leiocarpa (Vog.) Macbride (yvyra pere) secada en cámara solar. Investigación Agraria 9(2):72-77.

Dávalos, R. y G. M. Bárcenas. 1999. Clasificación de las propiedades mecánicas de las maderas en condición "seca”. Madera y Bosques 5(1):61-69.

De la Paz-Pérez, C. y A. Quintanar. 2001. Características anatómicas de la madera de Quercus castanea Née, Q. crassifolia Humb. et Bonpl. y Q. laurina Humb. et Bonpl. Revista Ciencia Forestal en México 24 (85):95-115.
De la Paz-Pérez, C. y R. Dávalos. 2008. Algunas características anatómicas y tecnológicas de la madera de 24 especies de Quercus (encinos) de México. Madera y Bosques 14(3):43-80.

De la Paz-Pérez, C., R. Dávalos y A. Quintanar. 2005. Influencia de los radios en algunas propiedades físicas y mecánicas de la madera de ocho encinos (Quercus) de Durango, México. Madera y Bosques 11(2):49-68.

De la Paz-Pérez, C., S. Vélez y J. Ceja. 2006. Anatomía de la madera de ocho especies de Quercus (Fagaceae) de Oaxaca, México. Madera y Bosques 12(1):63-94.

Flores, R., M.E. Fuentes, J. Quintanar y J.C. Tamarit. 2013. Maquinado de cuatro especies maderables de encino de la sierra de Juárez, Oaxaca. Revista Mexicana de Ciencias Forestales 4(16):22-33.

Flores, R., J.V. Rangel, J. Quintanar, M.E. Fuentes y L. Vázquez. 2007. Calidad de maquinado de la madera de Quercus affinis y Quercus laurina. Revista Chapingo Serie Ciencias Forestales y del Ambiente 13(1): 41-46.

Franklin, G.L. 1946. A rapid method of softening wood for microtome sectioning. Tropical Woods 88:35-36.

González, A., D.M. Arias, S. Valencia y K. Oyama. 2004. Morphological and RAPD analysis of hybridization between Quercus affinis and Q. laurina (Fagaceae), two Mexican red oaks. American Journal of Botany 91(3):401-409.

Honorato, J.A. 2002. Características anatómicas de la madera de encino. In: O.J. Quintanar, ed. Características, propiedades y procesos de transformación de la madera de los encinos de México. Inifap-CIRCE. C. E. San Martinito. Puebla, México. p:34-68.

Honorato, J. A. y J. Hernández. 1998. Determinación de componentes químicos de la madera de cinco especies de encino del estado de Puebla. Madera y Bosques 4(2):79-93.

Honorato, J. A. y M.E. Fuentes. 2001. Propiedades físico-mecánicas de la madera de cinco especies de encino del estado de Guanajuato. Revista Ciencia Forestal en México 26(90):5-28.

IAWA Committee. 1937. Standard terms of length of vessel members and wood fibers. Tropical Woods 51:21-22.

IAWA Committee. 1939. Standard terms of size for vessel diameter and ray width. Tropical Woods 59:51-52. 
IAWA Committee. 1989. List of macroscopic features for hardwood identification. IAWA Bulletin 10(3):219-332.

Kremer, A., A.G. Abbott, J.E. Carlson, P.S. Manos, C. Plomion, P. Sisco, M.E. Staton, S. Ueno y G.G. Vendramin. 2012. Genomics of Fagaceae. Tree Genetics \& Genomes 8(3):583-610.

Metcalfe, C.R. y L. Chalk. 1985. Anatomy of the dicotyledons: Wood structure and conclusion of the general introduction. Clarendon Press. Oxford. 297 p.

Montaño, S.A., S.L. Camargo y C. de la Paz-Pérez. 2013. Ecoanatomía de los elementos de vaso de la madera de cinco especies del género Mimosa (Leguminosae-Mimosoideae). Botanical Sciences 91(1):1-10.

Munsell Color Company. 1975. Munsell soil color charts. Baltimore, Maryland. 17 p.

Nájera, J.A., Z. Vargas, J. Méndez y J.J. Graciano. 2005. Propiedades físicas y mecánicas de la madera en Quercus laeta Liemb. de El Salto, Durango. Ra Ximhai 1(03):559-576.

Nixon, K.C. 1993. The genus Quercus in Mexico. In: T.P. Ramamoorthy, R. Bye, A. Lot, y J. Fa, eds. Biological diversity of Mexico: origins and distribution. Oxford University Press. Nueva York, EUA. p:447-458.

Oh, S.H. y P.S. Manos. 2008. Molecular phylogenetics and cupule evolution in Fagaceae as inferred from nuclear CRABS CLAW sequences. Taxon 57(2):434-451.

Ortega, F., L. Guerrero, T. Carmona y C. Córdoba. 1988. Angiospermas arbóreas de México. Núm. 1. Anatomía de la madera de 28 especies de Cosautlán de Carvajal, Veracruz. Boletín Técnico La Madera y su Uso No. 19. Instituto Nacional de Investigaciones sobre Recursos Bióticos-Universidad Autónoma Metropolitana Atzcapotzalco. México. D.F. 206 p.

Quintanar, J. 2002. Características, propiedades y procesos de transformación de la madera de los encinos de México. Inifap-CIRCE. C. E. San Martinito. Puebla, México.

Ramos, C.H. y V. Díaz. 1981. Instrucciones para recolectar muestras de madera para estudios tecnológicos. Instituto Nacional de Investigaciones Forestales. Boletín Divulgativo.

Ruiz-Aquino, F., J.I. Valdez, F. Manzano, G. Rodríguez, A. Romero y M.E. Fuentes. 2014. Ecuaciones de biomasa aérea para Quercus laurina y Q. crassifolia en Oaxaca, México. Madera y Bosques 20(2):33-48.

Ruiz-Aquino, F., J. I. Valdez, A. Romero, F. Manzano y M. E. Fuentes. 2015a. Spatial distribution of two oak species and ecological attributes of pine-oak woodlands from Ixtlán de Juárez, Oaxaca. Revista Chapingo Serie Ciencias Forestales y del Ambiente 21(1):67-80.

Ruiz-Aquino, F., M.M. González-Peña, J.I. Valdez, U.S. Revilla y A. Romero. 2015b. Chemical characterization and fuel properties of wood and bark of two oaks from Oaxaca, Mexico. Industrial Crops and Products 65:90-95.

Rzedowski, J. 1978. Vegetación de México. Limusa. México. $432 \mathrm{p}$.

Sandoval, E. 2005. Técnicas aplicadas al estudio de la anatomía vegetal. Cuadernos del Instituto de Biología 38. UNAM. México, D.F. 281 p.

SAS Institute. 1989. SAS user's guide statistics. Cary, North Carolina. SAS Institute Inc.

Sotomayor, J.R. 2005. Características mecánicas y clasificación de la madera de 150 especies mexicanas. Investigación e Ingeniería de la Madera 1(1):1-24.

Tortorelli, L. 1956. Maderas y bosques argentinos. ACME. Buenos Aires. 910 p.

Tovar, E. y K. Oyama. 2004. Natural hybridization and hybrid zones between Quercus crassifolia and Quercus crassipes (Fagaceae) in Mexico: morphological and molecular evidence. American Journal of Botany 91(9):1352-1363.

Valencia, S. 2004. Diversidad del género Quercus (Fagaceae) en México. Boletín de la Sociedad Botánica de México 75:33-53.

Valencia, S., M. Gómez. y F. Becerra. 2002. Catálogo de encinos del estado de Guerrero, México. Libro Técnico No. 1. Instituto Nacional de Investigaciones Forestales, Agrícolas y Pecuarias. México. 180 p.

Manuscrito recibido el 17 de mayo de 2014. Aceptado el 10 de noviembre de 2015.

Este documento se debe citar como:

Ruiz-Aquino, F., M.M. González-Peña, J.I. Valdez-Hernández y A. Romero-Manzanares. 2016. Estructura anatómica de la madera de dos encinos de Oaxaca. Madera y Bosques 22(1):177-189. 